\title{
EL CAMINO DE LA INCLUSIÓN DE PERSONAS CON NECESIDADES EDUCATIVAS ESPECIALES EN COSTA RICA: APORTES PARA LA DISCUSIÓN
}

\author{
Viviana María Richmond González \\ Docente de Educación Especial del Equipo Regional Itinerante \\ Dirección Regional de Educación de Heredia \\ Ministerio de Educación Pública
}

Recibido 14-IX-2009 • Aceptado 22-X-2009 • Corregido 11-XI-2009

"Yo soy solamente si tú también eres"

Desmond Tutu

Resumen: El presente ensayo esboza una serie de reflexiones acerca del estado actual de la Educación Especial, tomando en consideración los aciertos y los factores coexistentes que debilitan y anulan el proceso hacia la educación inclusiva. Examina, dentro del ámbito de la Educación Especial, el papel de determinadas(os) funcionarias(os) y sus aportes individuales, la influencia del sistema ideológico imperante y la forma en que los proyectos de este tipo de educación han sido desarrollados en Costa Rica. Su objetivo es constituirse en un aporte a esta amplia discusión nacional hacia el esclarecimiento de metas comunes y operativas, con el propósito de tener un sistema educativo realmente inclusivo.

Palabras clave: Educación especial, diversidad, inclusión.

\section{Introducción}

Uno de los mayores retos que actualmente enfrenta la Educación Especial, en conjunto con otras disciplinas, es hacer realidad una educación para todas las personas; es decir, una educación enfocada hacia el respeto de la diversidad social y la inclusión ${ }^{1}$ de cada ser humano en su carácter único e irrepetible que enriquece, desde sus particularidades, el currículo escolar (Arnaiz, 2004; Arnaiz, 2005; Arnaiz, s.f.; Blanco, 2005; Echeita, 2006; Hegarty, 2006; Marchesi, 2006; Martín, 2006; Meléndez, 2001; Moreira, 2008; Organización de las Naciones Unidas para la Educación, la Ciencia y la Cultura [UNESCO], 2004; UNESCO y Proyecto Regional de Educación para América Latina y el Caribe [PRELAC], 2007; Verdugo, s.f.). En este sentido, el Temario 


\begin{abstract}
The present essay poses a series of reflections regarding the current state of Special Education, considering the well-made decisions along with the coexistent factors that weaken and annul the process towards an inclusive education. It examines the role of specific employees and their individual contributions, as well as the influence of the prevailing ideological system, special education, and the way in which projects related to this topic have been developed in Costa Rica. The objective is to contribute to this wideranging national discussion in order to understand more clearly what our common goals and strategies should be, in the interest of achieving a truly inclusive educational system.
\end{abstract}

Key words: Special education, diversity, inclusion.
Abierto sobre Educación Inclusiva indica que "es necesario generar una corriente de opinión a favor de la inclusión y comenzar un proceso que busque consenso desde sus primeras etapas" (UNESCO, 2004, p. 28).

De este modo, cuando se tiene una meta común, lo primordial es tratar de esclarecer el camino. En Costa Rica, este camino se ha iniciado desde hace ya algunos años con importantes aciertos, pero también con grandes desaciertos.

En el presente artículo se abordarán de forma crítica diferentes acciones que ha desarrollado el Estado Costarricense para acercarse operativamente a la educación inclusiva, con la intención de valorar sus alcances y aportar a la reflexión nacional en la búsqueda de consensos y de propuestas más válidas y funcionales.

En la medida en que cada uno de los elementos vinculantes con la educación inclusiva sea discutido, analizado y mejorado, la meta final puede emerger con mayor claridad: la concreción de una sociedad más justa, solidaria y equitativa.

Por otra parte, es importante anotar que tanto las referencias a ideas expresadas por otras personas que se mencionan en el texto, así como los ejemplos usados, son extraídos fundamentalmente de la propia experiencia laboral de la autora, la cual incluye puestos de docencia en Educación Especial, la dirección de un Centro de Educación Especial, la participación en el Equipo Regional Itinerante ${ }^{2}$ y como asesora en el ámbito nacional. Asimismo, cabe anotar que la autora ha recopilado y transcrito estos testimonios.

\section{Los aciertos}

La historia de la Educación Especial ha evidenciado el esfuerzo reiterado de padres, madres y profesionales por la reivindicación de los derechos de las personas con discapacidad (Verdugo, s.f.). En este proceso, los aciertos están relacionados 
con los esfuerzos que han realizado ciertos sectores en todas las áreas y niveles de atención de la población con discapacidad.

Enelámbito nacional no se pone en duda, por ejemplo, el apoyo dado a la promulgación de la Ley 7600: Igualdad de Oportunidades para las Personas con Discapacidad por parte de Rossete Kleiman, Álvaro Mendieta, Ana Helena Chacón, Mildred García y el Dr. Alfaro, así como del Consejo Nacional de Rehabilitación y Educación Especial y la Defensoría de los Habitantes. De igual manera, se rescata la claridad conceptual de la Dra. Lady Meléndez, asesora nacional durante muchos años en el Departamento de Educación Especial y quien contó en su momento con los permisos del Ministerio de Educación Pública (MEP) para realizar todos sus estudios e investigaciones. Asimismo, fue fundamental la contribución de la M.Sc. Bárbara Holst, quien presidió durante algunos años el Consejo Nacional de Rehabilitación y Educación Especial. En representación del personal docente, cabe mencionar el trabajo realizado por la Licda. Ana Lucía Barrantes, docente en el Instituto Andrea Jiménez que actualmente labora en el Centro de Enseñanza Especial de Heredia, donde padres y madres luchan para que sea ella quien les imparta las lecciones a sus hijos e hijas.

No se pretende ser exhaustivo en este artículo con la lista de personas citadas anteriormente que han aportado gran riqueza a la Educación Especial con su visión humanista; el interés radica, más bien, en recalcar que una docente con entrega, mística y objetividad, una directora responsable en la ejecución de sus funciones, una asesora que ofrece un verdadero apoyo y denuncia situaciones irregulares, y, en general, cualquier persona que trabaje con ética en sus funciones son quienes han hecho y seguirán haciendo la diferencia.

Sin embargo, es fundamental aclarar que el panorama de trabajo de estas personas no necesariamente era ni es el más apropiado. Según Marcuse (1964), las personas que trabajan en una institución del Estado y que se caracterizan por su efectividad y eficiencia son sometidas a altas presiones sociales derivadas del sistema ideológico imperante.

Dussel (1999, citado por Hinkelammert y Mora, 2009) indica en este sentido que "se trata entonces de una totalización de la institución, de una fetichización, de una autorreferencia que niega la vida humana a favor del sistema.... La ley del sistema como tal... se convierte en la última instancia" (p. 400).

Con respecto a este tema, Berger (1999) señala que a medida que las personas van aceptando las normas y rutinas establecidas por las instituciones, se someten a los intereses sociales impuestos mediante el ejercicio del poder. Por ello, se implementan una serie de métodos para "poner en línea a sus miembros", los cuales varían de una situación social a otra y pueden ir desde la murmuración hasta la aplicación de leyes.

\section{Los obstáculos}

Si bien existen aciertos que deben ser tomados en cuenta, parece que desde hace muchos años los obstáculos cayeron como piedras enormes y ha sido difícil removerlos. Algunas de estas piedras solamente han cambiado de lugar, otras se han erosionado y existen las que se han empezado a formar con nuevas dimensiones. Aunque ciertos sectores de la sociedad costarricense reconocen muchos de estos obstáculos, es recurrente encontrar una actitud de indiferencia ante esta realidad.

A continuación se enumeran algunos de los principales obstáculos que se identifican en un camino hacia la educación inclusiva.

\section{Ideología social basada en un sistema capitalista neoliberal}

El tipo de ideología que impera en toda sociedad es la base que determina la posibilidad de la existencia de las personas 
que la integran, sus estructuras y relaciones (García, 2007, Hinkelammert y Mora, 2009, Marcuse, 1964). En este sentido, no es posible obviar que "el individuo es un elemento de la estructura familiar; la familia, por su parte, es un elemento formador de la estructura social y, a la vez, la estructura social modela a la familia y al propio individuo" (Campabadal, 2008, p. 66). El análisis del sistema desde un punto de vista inclusivo dirige su mirada a determinar en qué medida este favorece o no la presencia de relaciones justas, equitativas y solidarias entre los seres humanos.

De acuerdo con Hinkelammert y Mora (2009), la ideología actual se encuentra basada en el capitalismo neoliberal, el cual se caracteriza por la búsqueda de fines mercantilistas que benefician directamente a determinada clase social. Esta lógica capitalista, que por lo general no resulta tan lógica para la mayoría de la población (Marcuse, 1964), es incesantemente invisibilizada desde cualquier propuesta dirigida a la atención de personas con necesidades educativas especiales asociadas a la discapacidad y a los diferentes grupos llamados "minoritarios".

En este sentido, la mayoría de grupos sociales han considerado que el sistema es inevitable y que se debe aceptar sin ser cuestionado, cuando, por el contrario, se refiere a "un proceso histórico desigual entre los seres humanos, un localismo que se expande y generaliza por el resto de culturas" (Sánchez, citado por Campabadal, 2008, p. 44).

Flores (citado por Campabadal, 2008) indica que las diferentes clases sociales en ascenso formulan sus pretensiones en nombre de la humanidad mediante una ideología hegemónica. Dicha minoría se ha encargado de construir la realidad oficial en función de sus propios intereses y ha cuestionado todo aquello que se oponga a su manera de interpretar la realidad.

Desde esta lógica, es necesario desarrollar en los grupos poblacionales que no pertenecen a la clase social privilegiada las actitudes de sumisión, conformismo y represión con el fin de que no se lleven a cabo cuestionamientos sobre el porqué y el cómo de su existencia (García, 2006). Dentro de esta maquinaria, el sistema educativo público es garante de dicha ideología (Díaz, 2003), ya que estos(as) futuros(as) obreros(as) requieren asistir a un sistema educativo que mancille sus intenciones de analizar, criticar y denunciar las acciones de irrespeto a sus derechos. Todo esto se realiza por medio de un muy bien estructurado "sistema de evaluación" acompañado de un sólido currículo oculto que expulsa a quienes no se adaptan. Al respecto, el Dr. Leonardo Garnier Rómulo, Ministro de Educación Pública, indicaba al iniciar su gestión en el año 2004 que existía una relación directamente negativa entre las oportunidades laborales bien remuneradas y las personas que solo contaban con el título de Primaria.

Gehlen (citado por Berger, 1999) afirma que:

Las instituciones, son el equivalente artificial al instinto animal encargado de canalizar la acción humana hacia los parámetros impuestos por la sociedad. Dicha canalización se lleva a cabo mediante la formulación de opciones que se presentan como únicas posibles para el individuo como elemento social (p. 13).

Ante esta realidad, las personas con discapacidad generalmente "no son ajustables" al sistema, el cual las desprestigia mediante la lástima y las aísla en sistemas segregados (aulas integradas, centros de educación especial), simplemente porque se las considera poco competitivas y rentables en términos económicos.

En este sentido, García (2007) establece que "la globalización no sólo homogeniza e integra a las culturas. También genera procesos de estratificación, segregación y exclusión" (p. 221). Del mismo modo, Díaz (2003) indica que el proceso de globalización está ajustado al consumismo y a la uniformidad: "dentro de esta visión, quien no está en ese 'planeta', simplemente no está" (p. 21). Al respecto, Gallardo (2008) sostiene que todas aquellas personas 
que no pueden ser dominadas socialmente desde la actual ideología se incluyen dentro del grupo que debe ser eliminado.

Campabadal (2008) indica, por su parte, que las desigualdades que genera el actual sistema social se sostienen por una fórmula simbólica donde "diferente = inferior/peligroso". Por ello, la desigualdad y la discriminación se retroalimentan por medio de la producción social de los diferentes tipos de consenso que las legitiman.

Ante esta realidad, Assmann (1995 citado por Hinkelammert y Mora, 2009) señala que "el hecho mayor de la coyuntura actual del mundo es ciertamente el imperio pavoroso de la lógica de la exclusión y la creciente insensibilidad de muchísimos en relación a ella" (p. 401). Bajo este panorama, aspectos como la competitividad y la producción económica adquieren un alto valor, siendo el tiempo de producción de la mercancía uno de los elementos más importantes en la organización del trabajo (Santos de Morais, 1980).

Marcuse (1964) menciona al respecto que "el aparato impone sus exigencias económicas y políticas para la expansión y defensa sobre el tiempo de trabajo y el tiempo libre" (p. 25). Bajo este esquema de organización, "los excluidos son los no-competitivos de la sociedad" (De Souza, 2009, p. 2). Por lo tanto, las personas con discapacidad representan un obstáculo para la ideología imperante.

Por su parte, Palacios (2008), al referirse a los efectos de la Revolución Industrial -cuna del capitalismo- en las personas con discapacidad, indica:

El ser humano adquiere una dimensión económica determinada por su capacidad de producción, todo aquel sector de la sociedad que tenía alguna discapacidad quedó sujeto a la dimensión productiva, en la cual, dentro de los conceptos de dominación y dependencia características del capitalismo, su incorporación a la sociedad productiva se hacía en proporción directa a su capacidad de generar riquezas (p. 4).

Sin embargo, la realidad en la que vivían las personas con discapacidad posterior a la Revolución Industrial puede considerarse una constante ante la profundización del actual sistema neoliberal. Hinkelammert y Mora (2009) afirman al respecto:

Para ésta (la economía dominante neoclásica), la racionalidad formal abstracta (eficiencia, rendimiento, utilidad, competitividad, maximización, equilibrios macroeconómicos, etc.), se ha transformado en la "substancia", en el valor supremo y el fin en sí mismo, en referencia al cual la vida humana real se puede producir o no. La producción tiene que ser ante todo, lo más eficiente posible, máxima, competitiva; para sólo después considerar y decidir cuántos y quiénes pueden vivir a partir de este resultado. Y esto no excluye la necesidad de un "cálculo de vidas" (p. 44).

Posterior al análisis realizado al sistema económico imperante, los autores antes citados establecen que la sociedad se debería dirigir hacia una economía que respete la vida: "Otro mundo es posible es el mundo en el cual quepan todos los seres humanos.... Se trata, además, de la concepción de un mundo en el cual quepan diferentes culturas, naciones, razas, etnias, géneros, preferencias sexuales, etc." (Hinkelammer y Mora, 2009, p. 403).

Mientras el sistema social tenga su base en una economía competitiva y no en una economía solidaria que reconozca los talentos y los esfuerzos de cada ser humano desde la diversidad y se dirija a la satisfacción de las necesidades básicas para la vida $\mathrm{y}$, por lo tanto, al respeto de sus derechos humanos, cualquier propuesta educativa dirigida hacia la inclusión no será más que una novela romántica que muchos(as) seguirán añorando en la realidad.

\section{Desarrollo de proyectos con poca planificación, seguimiento y sistematización}

El sistema capitalista se encuentra estrechamente ligado con la inoperancia, desactualización, burocratización y negligencia general que embiste a las 
instituciones del Estado (Hinkelammert y Mora, 2009) y, entre ellas, al sistema educativo.

Como parte de esta misma lógica social, son pocas las propuestas educativas que se realizan de forma planificada con una visión a largo plazo y de forma sistemática y operativa.

Ahumada, (citado por Castellano, 2004), indica al respecto que la planificación es un "instrumento destinado a permitir el más lúcido y completo análisis de prácticamente todos los complejos problemas que enfrenta la sociedad, y a ofrecer métodos de organización capaces de lograr la mejor utilización de los recursos con el mínimo posible de costo social" (p. 64).

Una adecuada planificación debe cumplir con las funciones de problematizar, explicar las situaciones problemáticas, diseñar alternativas y proporcionar el apoyo requerido para la ejecución y evaluación de las medidas tomadas (Pichardo, 1993).

Un ejemplo de la falta de planificación es la creación a finales del siglo pasado de los Equipos Regionales Itinerantes, los cuales nacen en la coyuntura conceptual entre el modelo de integración y el concepto de necesidades educativas especiales (G. Aguilar, comunicación personal, 17 de setiembre del 2009). Las compañeras que iniciaron este proceso dan fe de capacitaciones o inducciones impartidas desde las instancias nacionales; sin embargo, aún no queda claro si se realizó un diagnóstico previo como base para el planteamiento de la capacitación, ni si se efectuó una evaluación de impacto posterior a ella. Resulta interesante que, años después, en la investigación de Dobles, Navarro y Solano (2005), los resultados evidencian que no existe un manejo conceptual claro de lo que es la inclusión educativa en dichas instancias regionales.

Otro ejemplo se dio a inicios de la presente década. El director del Departamento de Educación Especial del Ministerio de Educación Pública de ese momento comentaba acerca del éxito de la inclusión en relación con el cierre de un porcentaje significativo de aulas integradas en el país. No obstante, no se consideró que esta situación también indicaba que no hubo variación en el flujo de estudiantes que se integraban de los Centros de Educación Especial a las aulas integradas. Al respecto, Blanco (2005) indica que desde la educación inclusiva:

La tendencia es que los centros de educación especial se transformen en centros de recursos y apoyo a la comunidad y a las escuelas comunes, y que sólo escolaricen a aquellos alumnos con necesidades educativas especiales muy significativas, aunque también existen experiencias en las que estos alumnos están en la escuela común (p. 2).

Tal parece que los pasos lógicos de todo proceso no son tomados en cuenta: diagnóstico, desarrollo de la propuesta a partir de los resultados de dicho diagnóstico, validación de la propuesta, ejecución, apoyo en la ejecución, seguimiento y evaluación final. Esta falta de visión profesional se justifica con el estribillo de "estamos contra el tiempo", y, por estar contra el tiempo, parece que los procesos se estancan en el inicio.

No deben existir hipótesis que se den por sentadas desde la suposición, por lo que se ofrecerán ejemplos desde vivencias particulares, tomados de la experiencia profesional acumulada de la redactora de este artículo, con los cuales se ponen en duda afirmaciones como:

Todas las asesoras y asesores nacionales tienen un adecuado manejo conceptual sobre inclusión. La autora ha escuchado a una asesora nacional expresarse de la siguiente manera: "Pobrecito, a este chiquito hay que ayudarlo". Esta frase tiene por sí misma una base profunda en el modelo de rehabilitación ${ }^{3}$.

- $\quad$ Todos(as) los(as) directores(as) de centros educativos comprenden la Ley 7600. Un director de un 
Centro Educativo en Heredia dijo literalmente: "Se debe recuperar a todoslosestudiantesque seencuentren académicamente enfermos". En un análisis rápido de la frase, se deduce que este director considera la discapacidad como una enfermedad y señala el deber de "recuperarla", en claro desconocimiento conceptual del término "discapacidad".

- El personal docente de Educación Especial trabaja desde la diversidady para la inclusión educativa. Aún con licenciaturas en Educación Especial, hay docentes que siguen expresándose de las siguientes formas: "Esa mamá está faltita", "Los de aula abierta, son como chapulincitos", "Qué pecado con esa niña”. Una docente del servicio de problemas emocionales y de conducta le preguntó a una estudiante que había reprobado tres veces el primer año: "Mamita, ¿usted sabe leer y escribir?" y se extrañó cuando la niña se puso a llorar posterior a su pregunta.

- A las docentes de I y II Ciclo ya se les ha dado demasiada formación sobre adecuaciones de acceso y curriculares. En otra institución y ante el análisis de las condiciones de una estudiante que requería apoyos permanentes a nivel académico, una docente de I Ciclo expresó: "Yo pensé que ella era buena". Prejuicios valorativos como "normal", "anormal", "buena" y "mala" siguen coexistiendo alrededor del tema de la discapacidad.

Estos ejemplos evidencian que los diferentes enfoques de atención a las personas con discapacidad coexisten, subyacen y siguen arraigados en las representaciones sociales de docentes, directores(as) y asesores(as) (Agencia de Cooperación Internacional del Japón y Consejo Nacional de Rehabilitación y Educación Especial, 2006 y Verdugo, s.f.). En este sentido, es importante tener claro que la promulgación de una política pública como la Ley 7600 no garantiza un cambio inminente en los modelos conceptuales desde los cuales se atiende a esta población (G. Aguilar, comunicación personal, 17 de setiembre del 2009).

Las teorías que se han planteado siguen siendo hipótesis; sin embargo, lo que se desea resaltar es la necesidad de realizar una investigación en Costa Rica que determine el estado actual de las personas con necesidades educativas especiales asociadas a la discapacidad y, en general, del proceso de inclusión educativa. En este sentido, Pichardo (1993) indica que el diagnóstico para la programación social tiene que ver con el "esclarecimiento de la naturaleza y magnitud de los problemas que se pretende atender y ubicación de los espacios estratégicos para actuar" (p. 69).

Hasta no tener información al respecto, toda iniciativa tiene la gran posibilidad de no reflejar a ciencia cierta la realidad sociocultural y, por lo tanto, de no responder a sus necesidades y características. En otras palabras, podría no poseer los elementos suficientes de validez y confiabilidad.

\section{Educación Especial a la deriva}

a. Perfil de los(as) docentes de educación especial y formación profesional

Cuando en el proceso de elección vocacional alguna persona decide estudiar Educación Especial, por lo general la reacción social ante ello es considerarla como "buena" porque ha optado por trabajar con personas "discapacitadas". Esta visión encuentra su lógica en el paradigma de rehabilitación, caracterizado por una visión de lástima hacia esta población (Agencia de Cooperación Internacional del Japón y Consejo Nacional de Rehabilitación y Educación Especial, 2006 y Verdugo, s.f.).

En términos operativos y laborales, esto podría implicar que si dicha población es 
integrada o incluida y por lo tanto atendida en el sistema educativo regular por un(a) docente de I y II Ciclo, se quebrantaría el imaginario colectivo alrededor de la persona que es de Educación Especial. Al respecto, Blanco (2005) indica que "el desarrollo de una educación inclusiva también implica un cambio importante en el rol de la educación especial" (p. 2).

Por su parte, la M.Sc. Gilda Aguilar, asesora nacional de Educación Especial, señala que en muchas ocasiones las personas que ponen más obstáculos al proceso de inclusión son los(as) mismos(as) docentes de Educación Especial (comunicación personal, 17 de setiembre del 2009).

Ainscow y Miles (2008) advierten que:

La educación especial puede ser una forma de ocultar la discriminación de que son víctimas algunos grupos de estudiantes bajo una calificación aparentemente inocente, que justificaría el bajo nivel de rendimiento $\mathrm{y}$, por lo tanto, la necesidad de un régimen educativo distinto (p. 26).

Cabe en este punto cuestionar los procesos de formación docente que las universidades, tanto estatales como privadas, han desarrollado, ya que a los diecisiete o dieciocho años, las personas pueden errar en la selección de sus carreras. En este sentido, se considera que es responsabilidad de los entes de formación orientar, no sólo para que la persona redescubra sus propias habilidades y las canalice en la carrera o profesión indicada, sino también en consideración de las otras personas que podrían recibir los servicios de quienes se sienten inconformes con lo que estudiaron. En esta realidad, muchas veces los resultados son catastróficos.

Asimismo, las universidades tienen aún mucho que revisar internamente. ¿Dónde están las evaluaciones de impacto del proceso de formación docente? ¿Pueden dar una garantía social de que su currículo realmente prepara para trabajar ante las tendencias actuales y desde los nuevos paradigmas y realidades socioculturales?
A lo mejor sus egresadas(os) podrían contribuir mucho a esta materia.

Blanco (2005) aporta en este sentido:

Si queremos que los docentes sean inclusivos y también capaces de educar en y para la diversidad es necesario que se produzcan cambios importantes en su propia formación. En primer lugar, las instituciones de formación docente deberían estar abiertas a la diversidad y formar docentes representativos de las distintas diferencias presentes en las aulas. En segundo lugar, se les debería preparar para enseñar en diferentes contextos y realidades, y en tercer lugar, todos los profesores, sea cual sea el nivel educativo en el que se desempeñen deberían tener unos conocimientos básicos, teóricos y prácticos, en relación con la atención a la diversidad, la adaptación del currículo, la evaluación diferenciada y las necesidades educativas más relevantes asociadas a las diferencias sociales, culturales e individuales (p. 3).

Es importante indicar en este punto que uno de los resultados de la investigación desarrollada por el Instituto de Desarrollo Profesional (IDP) del MEP sobre Necesidades de Capacitación reveló que solo un $36 \%$ de la población docente entrevistada era egresada de universidades privadas (Costa Rica. Ministerio de Educación Pública, 2008). Si se consideran estos datos, es inaceptable que las universidades públicas sigan responsabilizando únicamente a las universidades privadas de los grandes problemas existentes en nuestro país en la formación docente.

Al respecto, J. Pozo (comunicación personal, 20 de agosto del 2009) expresó en una reciente conferencia en la Universidad Nacional de Costa Rica que "el fracaso escolar no es que los alumnos deserten, sino que los que aprueban no aprendan".

b. Prácticas pedagógicas con una amplia tradición rehabilitadora

Siguiendo la línea de Ainscow y Miles (2008) respecto de las prácticas discriminatorias que se generan desde la Educación Especial, es necesario investigar los paradigmas que fundamentan las 
experiencias de aprendizaje que desarrollan dichos(as) profesionales. Una posibilidad sería mediante el análisis de sus planeamientos didácticos, en tanto cantidad de objetivos dirigidos a la eliminación de "conductas autoestimulatorias y disruptivas" en contraposición con los referidos al desarrollo de habilidades y destrezas.

La hipótesis de este artículo es que los planeamientos todavía reflejan no sólo elementos del paradigma de la rehabilitación, sino también la búsqueda por normalizar a un niño o niña, intentando en muchas ocasiones eliminar un comportamiento determinado porque molesta a la sociedad y no necesariamente porque afecte el desarrollo de las tareas de su vida cotidiana. Se violentan, en este sentido, los derechos humanos fundamentales relativos a la propia personalidad: el derecho a "ser" (Delors, 1994). G. Aguilar (comunicación personal, 17 de setiembre del 2009) agrega al respecto:

Prevalece la idea de la super especialización y el estatus que este proporciona frente a otros, propio del modelo rehabilitador. Parece que "intervenir" sobre "conductas autoestimulatorias y disruptivas" da una mejor idea de que se debe tener un alto grado de conocimiento en algo extremadamente particular. $\mathrm{El}$ otro asunto es que pareciera que los docentes de Educación Especial tienen más armas para intervenir, que para enseñar.

De la misma manera, Ainscow (citado por Ainscow y Miles, 2008) refiere:

La preocupación por las respuestas individualizadas, que han sido una característica de la educación especial, sigue desviando la atención de la creación de formas de enseñanza que puedan llegar a todos los educandos de una clase y del establecimiento de condiciones en las escuelas que fomenten esa evolución (pp. 25-26).

Estos mismos autores también señalan que las escuelas inclusivas no se logran traspasando las prácticas que se han desarrollado desde la Educación Especial a las instituciones de I y II ciclos, ya que es necesario generar nuevas propuestas que pasen del marco de planificación individualizado a una perspectiva que procure "personalizar la enseñanza por medio de un compromiso con toda la clase" (Ainscow, citado por Ainscow y Miles, 2008, p. 26).

\section{c. Desvalorización del aporte de otras(os) profesionales}

Desde esta perspectiva de manejo del poder proveniente del modelo rehabilitador, es común que muchos de los procesos de educación inclusiva estén a cargo únicamente de profesionales de la Educación Especial, o al menos liderados por ellos(as). Si bien la Educación Especial y la inclusión están estrechamente relacionadas, esta última debe considerarse una responsabilidad que engloba todas las esferas sociales (García, 2003). Blanco (2005) explicita el respecto:

Hay que pensar en la creación de centros de recursos comunitarios que incluyan diferentes perfiles profesionales con funciones complementarias, ya que el apoyo de los docentes que provienen de la educación especial no es suficiente para atender plenamente la diversidad (p. 4).

Aunque la actual Comisión de Educación Inclusiva ha superado en parte esta visión al estar conformada por personal de diferentes departamentos de la División de Desarrollo Curricular del MEP, es imprescindible que se abra a la participación de expertos(as) más allá del ámbito de la Educación. Contar con el apoyo de profesionales en las áreas de las Ciencias Sociales, como la Psicología, Sociología, Planificación y Trabajo Social, puede enriquecer desde la diversidad el proceso de educación inclusiva, al mismo tiempo que favorece la visión de responsabilidad compartida.

Incluso, cualquier persona que estudie las implicaciones del paradigma de atención a la diversidad estará de acuerdo en que debería ser asumido como base para el desarrollo social de un país. 


\section{Consideraciones finales}

Algunas personas cercanas a las visiones psicoanalíticas compartirán la idea de que sólo existe aquello que es nombrado. En la cultura latinoamericana de doble moral proveniente del sistema capitalista, nombrar representa en algunas ocasiones una traición. Sin embargo, lo que no se nombra no se puede reflexionar, y menos aún mejorar.

Para llegar a lograr una sociedad realmente inclusiva, las fuerzas del Consejo Nacional de Rehabilitación y Educación Especial no deben dirigirse exclusivamente a ver si un bus tiene rampas o no; también su accionar debe encaminarse a analizar las políticas socioeconómicas que imperan y generar las luchas necesarias para favorecer una economía para la vida (Hinkelammert y Mora, 2009). Con la Ley 7600 las soluciones en materia de legislación nacional no han terminado: apenas comienzan.

Esinaceptable que se sigan escuchando por parte de asesoras(es), directoras(es) de centros de educación especial y personas en puestos de poder frases queilustran su manejo del tema desde el modelo de la rehabilitación y con un amplio desconocimiento de lo que implica la diversidad. Como mínimo, es necesario que se asuma la responsabilidad de una actualización hacia este personal con cursos de impacto en las prácticas profesionales. Además, es fundamental revisar los mecanismos para la selección del recurso humano que tiene el MEP, ya que actualmente la presentación del título es suficiente para asignar un puesto en propiedad, lo que implica que no se lleva a cabo ningún tipo de proceso de evaluación de competencias para ejercer la docencia.

Una persona no puede hacerle frente sola a una situación tan compleja, se requiere de un equipo transdisciplinario de seres humanos que trabajen con una visión clara y objetiva. Se precisan mujeres y hombres que no solo hayan revisado las motivaciones intrínsecas que conllevaron a que trabajaran en determinada área, sino que hayan rescatado objetivamente las limitaciones y las potencialidades de esa experiencia.

Es prioritario que quienes laboran en la División de Desarrollo Curricular del MEP se rodeen de expertas(os) internacionales con un amplio conocimiento teórico y práctico de las tendencias mundiales en materia de educación inclusiva. De esta forma, posterior a un análisis de la realidad costarricense se pueden contextualizar aquellas acciones y lecciones aprendidas de otros países o, en su defecto, construir nuevas propuestas. Su papel no está en dar charlas a las instituciones educativas, su papel consiste en proponer cambios a la política educativa desde un conocimiento profundo de la realidad nacional.

Es necesario que las universidades se caractericen por su profesionalismo, en el sentido de determinar si su currículo es realmente lo que pregonan: flexible, adaptado a la realidad y generador de conocimientos y habilidades.

En definitiva, es esencial que, tanto las diferentes instituciones mencionadas en este texto como cada una de las personas que están vinculadas con los derechos humanos, ejerzan su responsabilidad y movilicen el tema tal y como lo propone el Temario Abierto sobre Educación Inclusiva (UNESCO, 2004). Hay que hablar del tema, y hablar mucho para que la gente lo entienda, asimile y asuma.

La educación inclusiva también implica una responsabilidad personal: la forma de apropiación del conocimiento junto con las oportunidades para compartirlo; la manera de interrelacionarse con respeto a cada una de las opiniones y formas de ver la vida; el modo de dirigirse hacia el(la) otro(a), ni más arriba ni más abajo, y la visión que se tenga de sí mismo(a) en tanto ser único e importante en este engranaje social.

El camino es largo, pero si no se discute, si no se retoman los aciertos y las lecciones aprendidas, si no se es humilde en reconocer los desaciertos, se estará en 
esta maraña llamada Educación Especial por muchos años más, sin una luz clara al final del camino.

\section{Notas}

1 "La inclusión no es lugar, sino sobre todo una actitud y un valor que debe iluminar políticas y prácticas que den cobertura a un derecho tan fundamental como olvidado para muchos excluidos del planeta -el derecho a una educación de calidad-, y unas prácticas escolares en las que debe primar la necesidad de aprender en el marco de una cultura escolar de aceptación y respeto por las diferencias" (Echeita, 2006, p. 76).

2 El Equipo Regional Itinerante está conformado por profesionales con especialidades en áreas como Psicología, Sociología, Trabajo Social, Educación de I y II Ciclo y Educación Especial. Tiene como objetivo fundamental el apoyo a los estudiantes con discapacidad que se encuentran incluidos en el sistema educativo regular.

El modelo biológico o médico-rehabilitador surge entre las dos Guerras Mundiales y considera que las deficiencias y limitaciones de las personas constituyen un problema. Por lo tanto, la persona se convierte en paciente y es atendida por profesionales bajo un manto de superioridad, protección y lástima, con el fin de lograr maximizar las destrezas funcionales de la vida diaria y así conseguir un empleo remunerado (Agencia de Cooperación Internacional del Japón y Consejo Nacional de Rehabilitación y Educación Especial, 2006).

\section{Referencias bibliográficas}

Agencia de Cooperación Internacional del Japón y Consejo Nacional de Rehabilitación y Educación Especial. (2006). Rehabilitación en Costa Rica. Situación y perspectiva. San José, Costa Rica: Poder Judicial.

Ainscow, M., y Miles, S. (2008). Por una educación para todos que sea inclusiva: ¿Hacia dónde vamos ahora? Perspectivas, 38(1). Oficina
Internacional de Educación, Ginebra, Suiza. Consultado el 23 de octubre del 2009, de: http://www.ibe.unesco.org/ fileadmin/user_upload/Publications/ Prospects/Prospects145_spa.pdf

Arnaiz, P. (2004). La educación inclusiva: Dilemas y desafíos. Educación, Desarrollo y Diversidad, 7(2), 25-40. Asociación Española para la Educación Especial. Consultado el 5 de julio del 2007, de: http:// www.aedes-nacional.com/revista7(2) articulo2.pdf

Arnaiz, P. (2005). Atención a la diversidad. Programación curricular. San José, Costa Rica: EUNED.

Arnaiz, P. (s.f.). Currículum y atención a la diversidad. Consultado el 5 de julio del 2007, de: http://www.usal. es/ inico/investigacion/jornadas/ jornada3/actas/conf2.pdf

Berger, P. (1999). Los límites de la cohesión social: Conflictos y mediaciones en las sociedades pluralistas. Barcelona: Galaxia Gutenberg.

Blanco, R. (2005). Los docentes y el desarrollo de escuelas inclusivas. Revista Proyecto Regional de Educación para América Latina y el Caribe, $\mathrm{N}^{\circ} 1,174$ 177. Oficina Regional de Educación para América Latina y el Caribe, Santiago, Chile. Consultadoel 2 deabril del 2009, de: http://unesdoc.unesco. org/images/0014/001446/144666s.pdf

Campabadal, M. (2008). Violencia y derechos humanos en mujeres emigrantes latinoamericanas residentes en Costa Rica. Tesis para optar por el grado de doctorado en Estudios Latinoamericanos. Facultad de Filosofía y Letras, Universidad Nacional de Heredia: Costa Rica. 
Castellano, H. (2004). Planificación: Herramientas para enfrentar la complejidad, la incertidumbre y el conflicto. Caracas: CENDES.

Costa Rica. Ministerio de Educación Pública. Instituto de Desarrollo Profesional. (2008). Necesidades de capacitación docente. San José, Costa Rica. Manuscrito sin publicar.

De Souza, J. (2009). Descolonizando la dicotomía del superior-inferior en la "idea de desarrollo" de lo universal, mecánico y neutral a lo contextual, interactivo y ético. Consultado el 2 de setiembre del 2009, de: http:// pensardenuevo.org/descolonizandola-dicotomia-del-superior-inferior-enla-idea-de-desarrollo/

Delors, J. (1994). Los cuatro pilares de la educación. En J. Delors (Comp.), La educación encierra un tesoro (pp. 91-103). México: UNESCO. Consultado el 2 de abril del 2009, de: http://148.202.105.241/biblioteca/ bitstream/20050101/946/1/Los_ cuatro_pilares_de_la_educacion.pdf

Díaz, D. G. (2003). Las rutas históricas de la globalización. San José, Costa Rica: Editorial de la Universidad de Costa Rica.

Dobles, C., Solano, S., y Navarro, T. (2005). Conociendo las realidades de los equipos regionales itinerantes en las veinte regiones educativas del pais. Informe final del proyecto de investigación. San José, Costa Rica: Fundación Mundo de Oportunidades. Centro Nacional de Recursos para la Inclusión Educativa (CENAREC).

Echeita, G. (2006). Educación para la inclusión o educación sin exclusiones. Madrid, España: Narcea.
Gallardo, H. (2008). Teoría crítica: Matriz $y$ posibilidad de derechos humanos. Murcia, España: David Sánchez Rubio Editor.

García, E. (2003). La formación de profesionales para la educación inclusiva. Montevideo. Consultado el 23 de octubre del 2009, de: http://www. oei.es/docentes/articulos/formacion_ profesionales_educacion_inclusiva_ teske.pdf

García, G. (2006). La posmodernidad y sus modernidades: Una introducción. San José, Costa Rica: Editorial de la Universidad de Costa Rica.

García, N. (2007). La globalización como historia de nuestro tiempo: Sociedad, cultura y economía. San José, Costa Rica: Editorial Librería Alma Mater.

Hegarty, S. (2006). Diversidad del currículo y necesidades especiales de educación. Revista Proyecto Regional de Educación para América Latina y el Caribe, $\mathrm{N}^{\circ}$ 3, 128-133. Oficina Regional de Educación para América Latina y el Caribe, Santiago, Chile. Consultado el 2 de abril del 2009, de: http://unesdoc.unesco.org/ images/0015/001516/151698s.pdf

Hinkelammert, F., y Mora, H. (2009). Hacia una economía para la vida (2da. ed.). San José, Costa Rica: Editorial Departamento Ecuménico de Investigaciones.

Marchesi, A. (2006). El valor de educar a todos en un mundo diverso y desigual. Revista Proyecto Regional de Educación para América Latina $y$ el Caribe, $\mathrm{N}^{\circ}$ 2, 54-69. Oficina Regional de Educación para América Latina y el Caribe, Santiago, Chile. 
Consultado el 2 de abril del 2009, de: http://unesdoc.unesco.org/ images/0014/001455/145502s.pdf

Marcuse, H. (1964). El hombre unidimensional: Ensayo sobre la ideología de la sociedad industrial avanzada. México: Editorial Joaquín Mortiz.

Martín, E. (2006). Currículo y atención a la diversidad. Revista Proyecto Regional de Educación para América Latina y el Caribe, $\mathrm{N}^{\circ} 3$, 112-119. Oficina Regional de Educación para América Latina y el Caribe, Santiago, Chile. Consultado el 2 de abril 2009, de: http://unesdoc.unesco. org/images/0015/001516/151698s.pdf

Meléndez, L. (2001). Sociología de la diversidad. San José, Costa Rica. Manuscrito sin publicar.

Moreira, T. E. (2008). Desafíos de la Ley 7600 ante las nuevas tendencias de la educación inclusiva. Revista Educación, 32(2), 57-71. Universidad de Costa Rica, San José. Consultado el 14 de abril del 2009, de: http:// redalyc.uaemex.mx/src/inicio/ArtP dfRedjsp?iCve $=44032205 \& \mathrm{iCveNu}$ $\mathrm{m}=10810$

Palacios, A. (2008). El modelo social de discapacidad: Orígenes, caracterización y plasmación en la Convención Internacional sobre los Derechos de las Personas con Discapacidad. Comité Español de Representantes de Personas con Discapacidad (CERMI).
Madrid, España: Grupo Editorial Cinca. Consultado el 17 febrero del 2009, de: http://www.cermi.es/ NR/rdonlyres/71F353B1-DDB9. 4217-A2C8-99F4D7876EBD/20212/ Elmodelosocialdediscapacidad2.pdf

Pichardo, A. (1993). Planificación y programación social. Buenos Aires: Editorial Humanitas.

Santos de Morais, C. (1980). Apuntes de teoría de la organización. Cuadernos de Capacitación. México D.F.: INCA RURAL.

UNESCO. (2004). Temario abierto sobre educación inclusiva. Santiago, Chile. Consultado el 22 de enero del 2008, de: http://portal.unesco.org/ es/files/21505/10886659511temario_ abierto_educacion_inclusiva_ manual.pdf/temario_abierto_educacion_inclusiva_manual.pdf

UNESCO y Proyecto Regional de Educación para América Latina y el Caribe [PRELAC]. (2007). Educación de calidad para todos: Un asunto de derechos humanos. Santiago, Chile. Consultado el 22 de enero del 2008, de: http://unesdoc.unesco.org/ images/0015/001502/150272s.pdf

Verdugo, M. A. (s.f.). La concepción de discapacidad en los modelos sociales. Consultado el 29 de octubre del 2006, de: http:// www.usal.es/ inico/publicaciones/Verdugo-ModelosSoc.pd 
